Copyright (C) 2008 IEEE.

Reprinted from the IEEE Radar Conference (2008 : Italy):pp. 1869-1874

This material is posted here with permission of the IEEE. Such permission of the IEEE does not in any way imply IEEE endorsement of any of the University of Adelaide's products or services. Internal or personal use of this material is permitted. However, permission to reprint/republish this material for advertising or promotional purposes or for creating new collective works for resale or redistribution must be obtained from the IEEE by writing to pubs-permissions@ieee.org.

By choosing to view this document, you agree to all provisions of the copyright laws protecting it. 


\title{
MIMO Noise Radar - Matched Filters and Coarrays
}

\author{
Douglas A Gray, Member, IEEE \\ EEE School, University of Adelaide \\ Adelaide Australia SA 5005 \\ phone: + (618) 8303 6425, fax: + (618) 8303 4360, email: dgray@eleceng.adelaide.edu.au \\ Amerigo Capria, Student Member, IEEE \\ Dept. of Information Engineering, University of Pisa \\ Via Caruso 16, 56122, Pisa, Italy \\ phone: + (39)0502217511, fax: + (39)0502217626, email: amerigo.capria@,iet.unipi.it
}

\begin{abstract}
The noise radar concept can be extended to an array of $K$ transmit antenna and $M$ receive antenna. When independent noise sources are transmitted from each antenna the approach may be viewed as a special case of MIMO radar and matched filters may be derived. In this contribution statistical properties of matched filters for MIMO noise radar are derived.

For any array the concepts of the sum and difference coarrays are useful and important tools for understanding an array's beamforming properties particularly for sparse arrays. Element space matched filters are shown to be related to the concept of the sum coarray and some examples of the advantages of this for sparse transmit/receive array geometry are provided. A variation of this result for beam space matched filters is also presented,
\end{abstract}

Index Terms-Noise radar, MIMO radar, Waveform diversity

\section{INTRODUCTION}

For single channel noise radar the use of a wide bandwidth and correlation processing enables range profiles with low sidelobes and suppressed range ambiguities to be realized [1]. Additionally there appear to be advantages in using noise waveforms in conjunction with low bit resolution analogue to digital convertors as intermodulation products are avoided. However perhaps the most motivating reason for the use of multi-channel noise radar is its low probability of intercept making such radars difficult to detect with standard ESM receivers [2]. A frequency domain formulation of the extension of single channel noise radar to multi-antenna on both transmit and receive indicated that two main transmission configurations

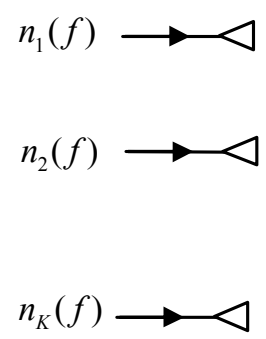

Figure 1: Element space
In the ES approach $\mathrm{K}$ channels of independent noise are transmitted by omni-directional antennas as illustrated in Figure 1.

For the BS approach proposed in [3] each independent noise source is used to form a beam illuminating a selected sector of the radar's field of view - effectively coding each sector according to a particular noise source. The direction of each sector is determined by the phase shifts and the width determined by the beam-width of the transmit array. The approach is illustrated in Figure 2 and it should be noted that the number of beams, $\mathrm{N}$, need not equal the number of transmit antennas but may be chosen to be less than $\mathrm{K}$.

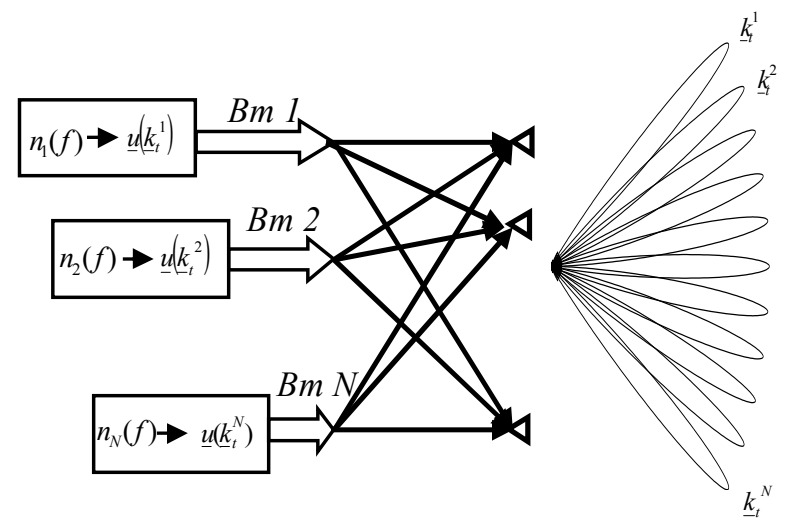

Figure 2: Beam Space transmit

In [3] matched filters for both the ES and BS approaches were derived and in this contribution statistical properties of ES matched filters are derived. For ES and the case of co-located transmit and receive arrays, a generalized form of the receiver matched filter leads to a beamforming scheme that is based on the sum coarray [5] of the transmit and receive arrays. The BS matched filter is also considered and shown to be equivalent to a hybrid beam/element space approach based on the sum coarray. 


\section{NOTATION AND BACKGROUND}

Let $\underline{x}_{t}(f)$ and $\underline{x}_{r}(f)$ denote the Fourier transforms of the transmit and receive antenna element waveforms respectively and let $\underline{n}(f)$ denote the Fourier transforms of a vector of independent noise sources. (The number of noise sources may be different for the ES and BS approaches.) Consider a single scatterer located as illustrated in Figure 3.

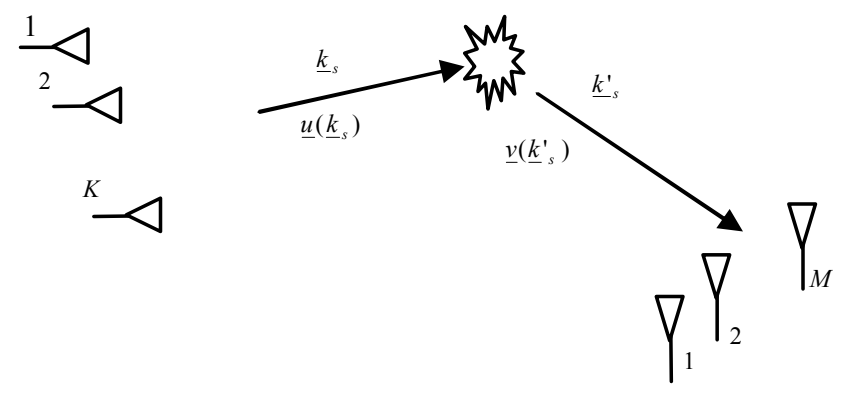

Figure 3: Transmit/receive geometry

In Figure $3 \underline{u}\left(\underline{k}_{s}\right)$ and $\underline{v}\left(\underline{k}_{s}^{\prime}\right)$ are the transmit and receive steering vectors and the wave-vectors $\underline{k}_{s}$ and $\underline{k}_{s}^{\prime}$ denote the direction of the scatterer from the phase centres of the $K$ element transmit and $M$ element receive arrays respectively.

For both ES and BS the matched filter corresponding to the scatterer illustrated in Figure 3 is given by

$$
\underline{h}_{m f}^{H}\left(\underline{k}_{s}, \underline{k}_{s}^{\prime}\right)=\frac{1}{\sigma_{n}} e^{j \phi_{s}} \underline{x}_{t}^{H}(f) \underline{u}\left(\underline{k}_{s}\right) \underline{v}^{H}\left(\underline{k}_{s}^{\prime}\right)
$$

where the phase delay, $\phi_{s}$ represents the time delay from the phase centre of the transmit array to that of the receive array via the scatterer and $\sigma_{n}$ is the transmit noise power.
The signal flow diagram of one implementation of the ES matched filter is illustrated in Figure 4 and illustrates how the matched filter, implemented at the receiver, incorporates both the transmit and receive beamforming.

\section{ES MATCHED FILTER}

For ES $\underline{x}_{t}(f)=\underline{n}(f)$ and the output of a filter matched to a scatterer in directions $\underline{\underline{k}}_{s}$ and ${\underline{\boldsymbol{k}^{\prime}}}_{s}$ w.r.t. the transmitting and receiving arrays respectively is given by

$$
y_{\text {mfes }}(.)=\underline{h}_{m f e s}^{H}(.) \underline{x}_{r}(f)=\frac{e^{j \phi_{s}} \underline{v}^{H}\left(\underline{\tilde{k}}_{s}^{\prime}\right)\left\{\underline{x}_{r}(f) \underline{n}^{H}(f) \underline{u}\left(\underline{\tilde{k}}_{s}\right)\right.}{\sigma_{n}}
$$

It is illustrative to compare the performance of the ES matched filter with that of a conventional beamformer at the receiver. To do this consider co-located receive and transmit arrays with the same phase centres. For this case $\underline{k}=\underline{k}_{s}=-\underline{k}_{s}^{\prime}$. (This is not an essential point - it just reduces the complexity of the notation.) The output of a conventional receive beamformer, $y_{\text {con }}(\underline{\tilde{k}})$, where $\underline{\tilde{k}}$ denotes the beam steering direction, is given by

$$
y_{\text {con }}(\underline{\tilde{k}})=\underline{v}^{H}(\underline{\tilde{k}}) \underline{x}_{r}(f) / M
$$

where $\underline{v}(\underline{\tilde{k}})$ is the steering vector. The output power of the conventional beamformer is given by

$$
P_{c o n}(\tilde{\tilde{k}})=\underline{\underline{v}}^{H}(\underline{\tilde{k}}) R_{x r}(f) \underline{v}(\underline{\tilde{k}}) / M^{2}
$$

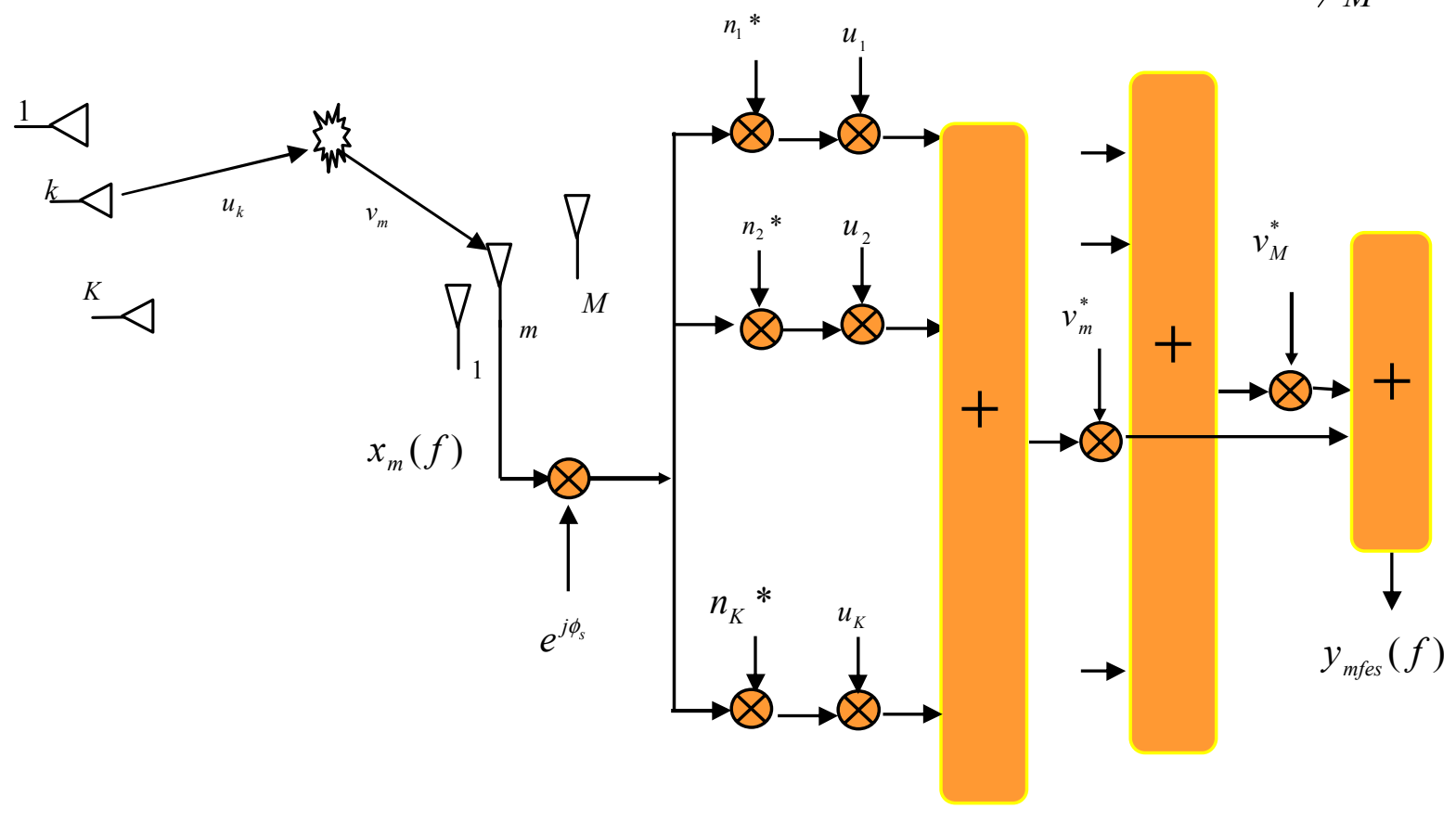

Figure 4: ES Matched filter implementation 
where $R_{x r}(f)$ is the cross-spectral matrix of the receiver outputs. For the case of a single scatterer with a normalized scattering cross-section of $\sigma_{s}^{2}$ located at a direction $\underline{k}$ from the array phase centre the above expression can be shown to reduce to

$$
P_{c o n}(\underline{\tilde{k}})=K \sigma_{s}^{2} \sigma_{n}^{2} \underbrace{\frac{\left|\underline{v}^{H}(\underline{\tilde{k}}) \underline{v}(\underline{k})\right|^{2}}{M^{2}}}_{\text {Beampattern of Rx array }}+\sigma_{w n}^{2} / M^{2}
$$

where $\sigma_{n}^{2}$ is the power of the transmit signal at any antenna and $\sigma_{w n}^{2}$ is the self noise (i.e., thermal noise) at a receiver.

Calculation of the output power of the matched filter is somewhat more complicated as both the matched filter and the received waveform contain the noise waveforms, see Eqn. 2. Thus it is first necessary to check whether the matched filter output has zero mean and in order to obtain analytic expressions it is first necessary to make the assumption that the transmit and receive waveforms are Gaussian.

From Eqn.2 and adding in self noise, the output of an ES filter matched to a scatterer in direction $\underline{\tilde{k}}$ from the array phase centre is given by

$$
\begin{aligned}
y_{\text {mfes }}(\tilde{\tilde{k}}) & =\underline{h}_{m f e s}^{H}(\underline{\tilde{k}})\left\{\underline{x}_{r}(f)+\underline{n}_{w n}(f)\right\} \\
& =\frac{\underline{n}^{H}(f) \underline{u}(\underline{\tilde{k}}) \underline{\nu}^{H}(\underline{\widetilde{k}})\left\{\underline{x}_{r}(f)+\underline{n}_{w n}(f)\right\}}{\sigma_{n}}
\end{aligned}
$$

For the single scatterer illustrated in Figure $3 \underline{x}_{r}(f)$ is given by

$$
\left.\underline{x}_{r}(f)=\sigma_{s} \underline{v} \underline{k}_{s}{ }^{\prime}\right) \underline{u}^{H}\left(\underline{k}_{s}\right) \underline{n}(f)
$$

and assuming, quite plausibly, that $\underline{n}(f)$ and $\underline{n}_{w n}(f)$ are uncorrelated, the expectation of both sides of the above expression reduces to

$$
E\left\{y_{m f e s}(\underline{\tilde{k}})\right\}=\frac{\sigma_{s}}{\sigma_{n}} E\left\{\underline{n}^{H}(f) \underline{u}(\underline{\tilde{k}}) \underline{\nu}^{H}(\underline{\tilde{k}}) \underline{v}\left(\underline{k}^{\prime} \underline{u}^{H}(\underline{k}) \underline{n}(f)\right\} .\right.
$$

The above expression may be rearranged to

$$
E\left\{y_{m f e s}(\underline{\tilde{k}})\right\}=\frac{\sigma_{s}}{\sigma_{n}} \operatorname{Tr}\left[\underline{u}(\underline{\tilde{k}}) \underline{v}^{H}(\underline{\tilde{k}}) \underline{v}(\underline{k}) \underline{u}^{H}(\underline{k}) E\left\{\underline{n}(f) \underline{n}^{H}(f)\right\}\right]
$$

and since $E\left\{\underline{n}(f) \underline{n}^{H}(f)\right\}=\sigma_{n}^{2} I$ the above expression can be shown to reduce to

$$
\left.E\left\{y_{m f e s}(\underline{\tilde{k}})\right\}=\sigma_{s} \sigma_{n}\left\{\underline{u}^{H}(\underline{k}) \underline{u}(\underline{\tilde{k}})\right\} \underline{v}^{H}(\underline{\tilde{k}}) \underline{v}(\underline{k})\right\}
$$

which is non-zero.

Interestingly

$$
\mid E\left\{y_{\text {mfes }}(\underline{\tilde{k}})\right\}^{2}=\sigma_{s}^{2} \sigma_{n}^{2} K^{2} M^{2} \underbrace{\frac{\left\{\underline{u^{H}}(\underline{k}) \underline{u}(\tilde{\underline{k}})\right\}^{2}}{M^{2}}}_{\text {Beampattern of Tx array Beampattern of Rx array }} \underbrace{\frac{\left\{\underline{v}^{H}(\tilde{k}) \underline{v}(\underline{k})\right\}^{2}}{K^{2}}}
$$

The above expression illustrates the ability of the matched filter to reconstitute, at the receiver, the beampattern of the transmit array.

An example is illustrative. Consider co-located transmit and receive linear arrays and a single scatterer at $-30^{\circ}$ whose normalized cross-section is $20 \mathrm{~dB}$. The expressions $P_{c o n}(\underline{\tilde{k}})$ and $\mid E\left\{y_{\text {mfes }}(\underline{\tilde{k}})\right\}^{2}$ are plotted in Figure 5 for the case where $K=8$ and $M=4$ and for both arrays $d / \lambda=0.5$. The ES matched filter results have been normalized to equal those of the conventional receive beamformer at their respective peaks.

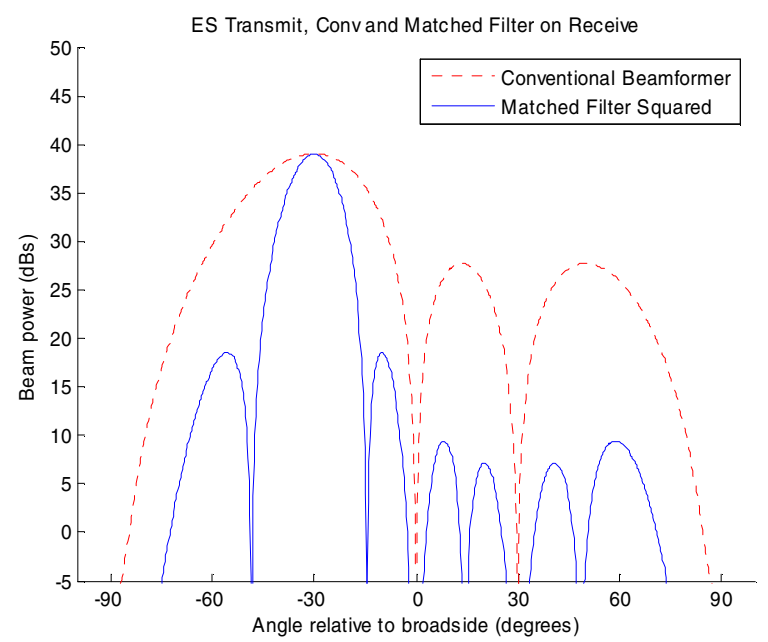

Figure 5: Single scatterer

In the above example the difference between the conventional beamformer and the ES matched filter can be clearly seen. The ES matched filter squared results do not depend on the level of white noise at the receiver and the effect of the transmit beam pattern can be clearly seen though the reduction of the width of the main lobe response due to the larger aperture of the transmit array. The above example can be extended to multiple scatterers and in Figure 6 results for two scatterers at $-30^{\circ}$ and $-10^{\circ}$ whose normalized radar crosssections are $20 \mathrm{~dB}$ and $10 \mathrm{db}$ respectively are plotted.

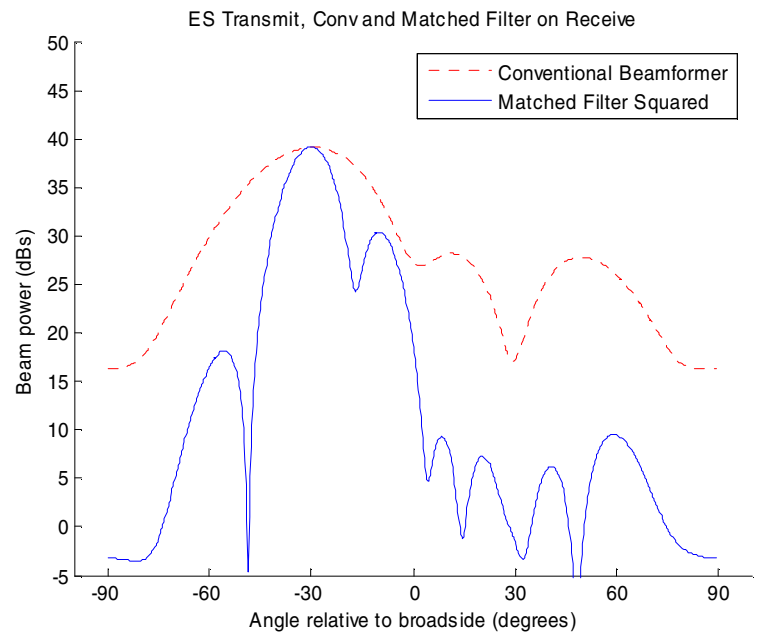

Figure 6: Two scatterers 
Again the advantage of the ES matched filter which takes into account the transmit antenna is well illustrated by improved angular resolution.

Evaluation of the variance of the matched filter output requires deriving an expression for

$$
\begin{aligned}
& E\left\{\left|y_{\text {mfes }}(\underline{\widetilde{k}})\right|^{2}\right\} \\
& =\frac{1}{\sigma_{n}^{2}} E\left\{\underline{n}^{H}(f) \underline{u}(\underline{\tilde{k}}) \underline{v}^{H}(\underline{\tilde{k}}) \underline{x}_{r}(f) \underline{x}_{r}^{H}(f) \underline{v}\left(\underline{k}_{)} \underline{u}^{H}(\underline{k}) \underline{n}(f)\right\}\right.
\end{aligned}
$$

For illustrative purposes consider the example of a single scatterer as illustrated in Figure 3. In this case the above expression reduces to

$$
E\left\{\left|y_{m f e s}(\underline{\tilde{k}})\right|^{2}\right\}=\frac{1}{\sigma_{n}^{2}} E\left\{\underline{n}^{H}(f) C \underline{n}(f) \underline{n}^{H}(f) C^{H} \underline{n}(f)\right\}
$$

where $C=\underline{u}(\underline{\tilde{k}}) \underline{v}^{H}(\underline{\tilde{k}}) \underline{v}(\underline{k}) \underline{u}^{H}(\underline{k})$ and after some tedious manipulation of fourth order Gaussian moments the above expression can be shown to reduce to

$$
E\left\{\left|y_{\text {mfes }}(\underline{\tilde{k}})\right|^{2}\right\}=\mid E\left\{y_{\text {mfes }}(\underline{\underline{k}})\right\}^{2}+\sigma_{n}^{2} \sigma_{s}^{2} \operatorname{Tr}\left\{C^{H} C\right\}
$$

Substituting for $\mathrm{C}$ and simplifying gives

$$
\operatorname{Tr}\left\{C^{H} C\right\}=K^{2}\left|\underline{v}^{H}(\underline{\tilde{k}}) \underline{v}(\underline{k})\right|^{2}
$$

Finally taking receiver noise into account the variance of the output of the matched filter can be shown to be given by

$$
\begin{aligned}
P_{m f e s}(\underline{\tilde{k}}) & =K^{2} M^{2} \sigma_{s}^{2} \sigma_{n}^{2} \frac{\left|\underline{v}^{H}(\underline{\tilde{k}}) \underline{v}(\underline{k})\right|^{2}}{M^{2}}+K M \sigma_{w n}^{2} \\
& =K M^{2} P_{\text {con }}(\underline{\tilde{k}})
\end{aligned}
$$

Thus the rather surprising conclusion that the variance of the matched filter is a scaled version of the output power of a conventional beamformer is obtained.

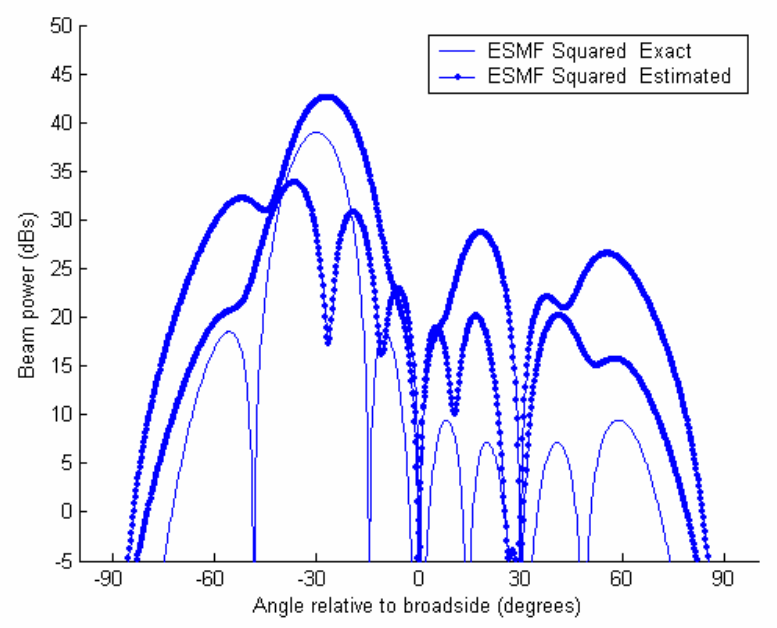

Figure 7: Two realisations
To illustrate the above single scatterer scenario was simulated in the frequency domain and the output of the ES matched filter for a single realization was calculated. Two realizations are illustrated in Figure 7 and they illustrate the large statistical variability of using just a single realization. The variance of single realization matched filter outputs was calculated for the above scenario using 100 independent realizations. This result is plotted in Figure 8 together with the expression for the output power of a conventional beamformer and illustrates close agreement with the result of Eqn (3) after the normalization factor $K M^{2}$ is accounted for.

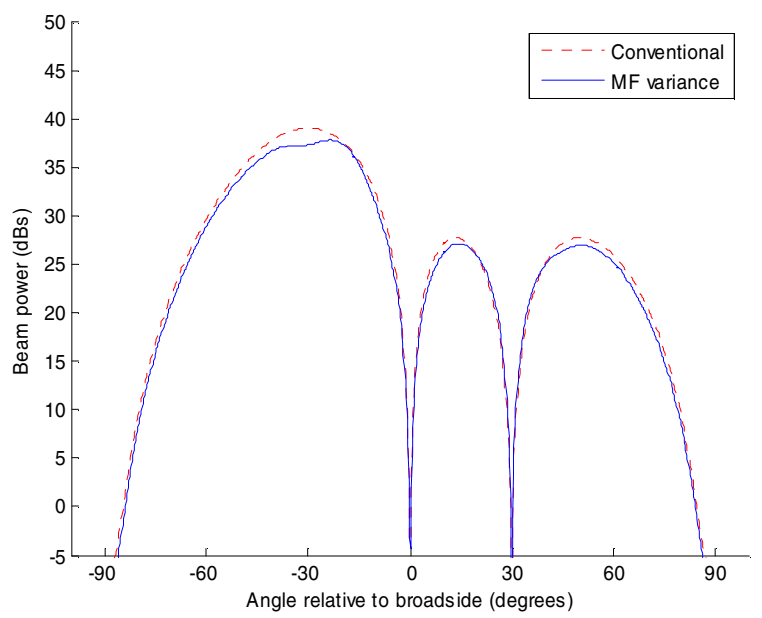

Figure 8: Matched Filter variance

The single realization results for the ES matched filter are typical of what would be obtained using a single pulse in the time domain. To improve the performance of the matched filter additional averaging of the matched filter outputs is required. Denoting $y_{m f e s}^{q}(\underline{\tilde{k}})$ as the $q$-th realisation the averaged output of the matched filter, i.e.,

$$
\frac{1}{Q} \sum_{q=1}^{Q} y_{m f e s}^{q}(\tilde{\tilde{k}})
$$

is plotted for various values of Q in Figure 9.

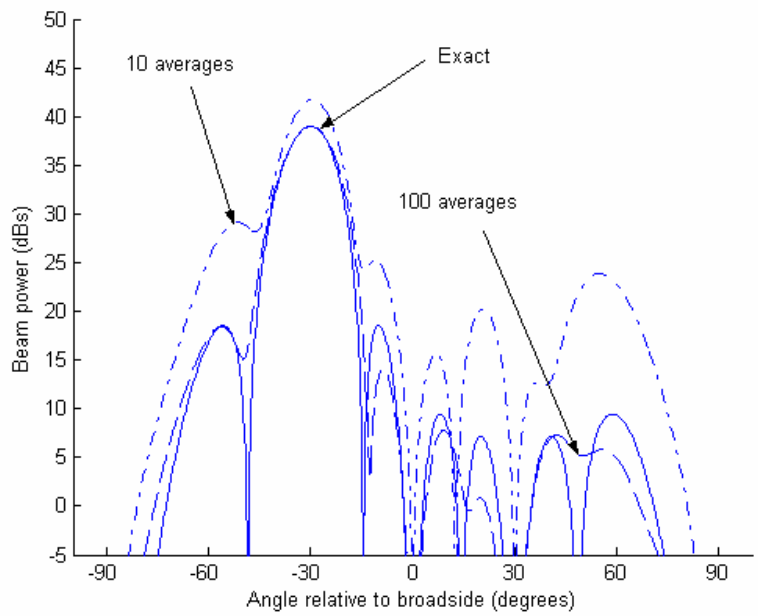

Figure 9: Averaging 


\section{Relationship of Matched Filter to COARRAys}

Under closer inspection of Figure 4 we can observe that output of each receive element, prior to multiplication by the transmit beamforming weights, can be decomposed into a further K channels through multiplication by the $n_{k}$.

Rewriting Eqn. 2 and ignoring the bulk time delay term, the matched filter output is given by

$y_{m f e s}(f)=\sum_{k=1}^{K} \sum_{m=1}^{M}\left\{\underline{u}\left(\underline{k}_{s}\right) \underline{v}^{H}\left(\underline{k}_{s}^{\prime}\right)\right\}_{k, m}\left\{\underline{x}_{r}(f) \underline{n}^{H}(f)\right\}_{m, k}$

In the above expression the phase terms can be rewritten as

$\left\{\underline{u}\left(\underline{k}_{s}\right) \underline{v}^{H}\left(\underline{k}_{s}^{\prime}\right)\right\}_{k, m}=e^{j \underline{k}_{s} \cdot \underline{d}_{k}} e^{-j \underline{k}^{\prime} \cdot \underline{d}_{m}}=e^{j\left(\underline{k}_{s} \cdot \underline{d}_{k}-\underline{k}_{s}^{\prime} \cdot \underline{d}_{m}\right)}$

Now consider co-located receive and transmit arrays with the same phase centres. For this case $\underline{k}_{s}=-\underline{k}_{s}^{\prime}$ and so the above expression reduces to

$$
\left\{\underline{u}\left(\underline{k}_{s}\right) \underline{v}^{H}\left(\underline{k}_{s}\right)\right\}_{k, m}=e^{j \underline{k}_{s} \cdot\left(\underline{d}_{k}+\underline{d}_{m}\right)}
$$

$000 * 000$
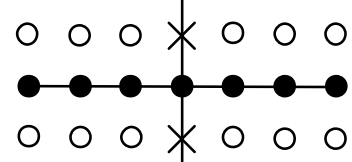

$000 * 000$

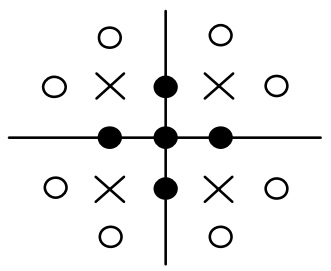

- Transmit array

$X$ Receive array

O Synthesized elements

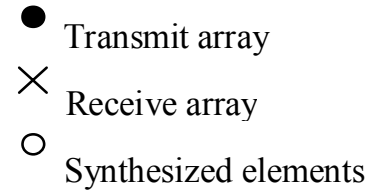

Figure 10: Coarray examples

These phase terms can be interpreted in term of a $K M$ element array as illustrated in Figure 11 with receivers located at the vector sum of the positions of the transmit and receive elements. Indeed this is just the sum coarray of the combined transmit and receive elements reviewed in [5]. Examples of some transmit/receive arrays and the resulting coarrays are given in Figure 10.
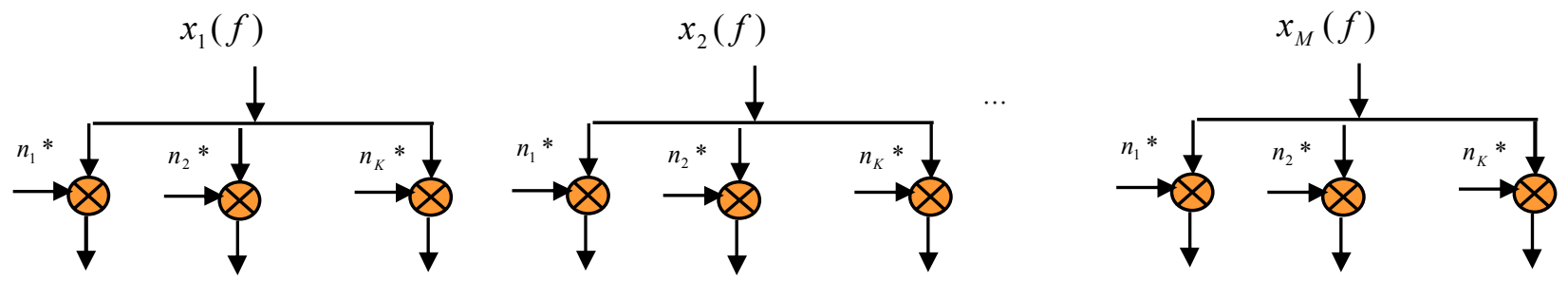

Figure 11: $K M$ synthesized outputs 


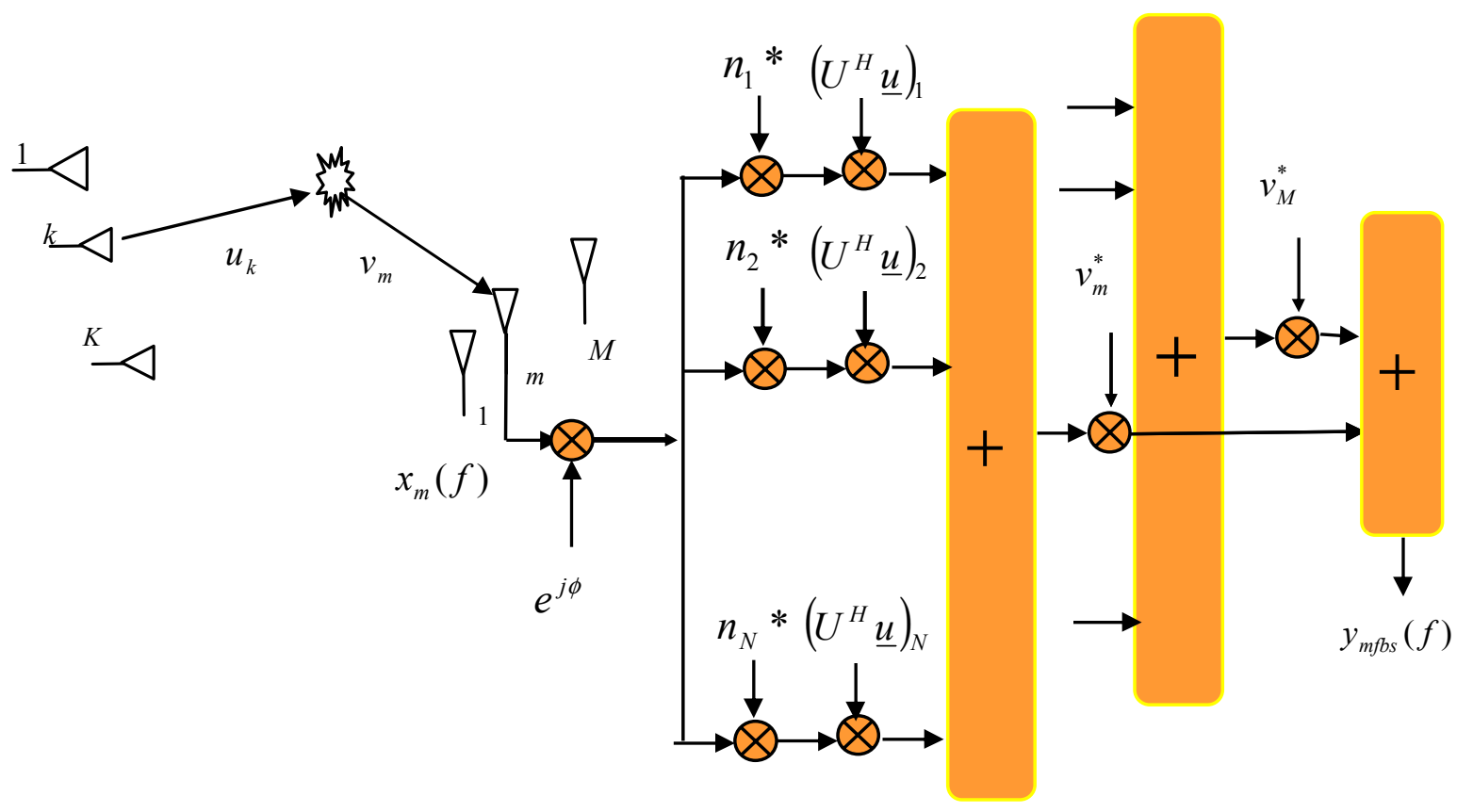

Figure 12: BS Matched filter implementation

\section{CONCLUSIONS}

MIMO noise radar can be formulated in terms of either element or beam space and matched filters have been given for each approach. Expressions for the mean output of the ES matched filter have been derived and shown to offer considerable improvement over a conventional beamformer using just the receiver outputs. However the instantaneous output of the ES matched filter has a large statistical variability and needs significant averaging before approaching the mean output. An expression for this statistical variability has been derived and confirmed by simulation.

In both cases the filters can be interpreted and implemented in terms of the coarray of the combined transmit/receive geometry. Whilst these virtual or phantom sensors offer conceptual advantages for conventional beamforming on both transmit and receive such implementations are not efficient and will not improve detection performance over simpler implementations of the matched filter. However the coarray approach does offer advantages in terms of DOA estimation procedures and allows the possibility of implementing MVDR processing on the coarray outputs.

\section{REFERENCES}

[1] S.R.J. Axelsson, "Noise Radar for Range/Doppler Processing and Digital Beamforming Using Low-Bit ADC", IEEE Trans. GeoSci. and Remote Sensing, December 2003, vol. 41, pp. 2703-2720

[2] D.A. Gray “Multichannel Noise Radar", International Radar Symposium, Krakow, Poland, 24-26 May 2006.

[3] D.A. Gray "Matched Filters for Multichannel Noise Radar", to appear in Defence Applications of Signal Processing Workshop , Fraser Island, Australia, December 2006.

[4] D.A. Gray and R. Fry "MIMO Noise Radar - Element and Beam Space Comparisons" Proc of WDD2007, Pisa, Italy, July 2007.

[5] R. T. Hoctor and S. A. Kassam, "The Unifying Role of the Coarray in Aperture Synthesis for Coherent and Incoherent Imaging," Proc IEEE, vol. 78, No. 4 pp. 735-752, April 1990.

[6] B.J. Donnet and I.D. Longstaff "MIMO Radar, Techniques and Opportunities" Proc of $3^{\text {rd }}$ European Radar Conference, Manchester, UK, September 2006.

[7] I. Bekkerman and J. Tabrikian "Target Detection and Localisation Using MIMO Radar and Sonar" IEEE Transactions on Signal Processing, vol. 54, No. 10 pp. 3873-3883, October 2006.

[8] L. Xu, J. Li and P. Stoica "Adaptive Techniques for MIMO Radar" Proc of $4^{\text {th }}$ IEEE Workshop on Sensor Array and Multichannel Processing, 249-253, July 2006. 